\title{
MAXIMAL AVERAGES ALONG CURVES OVER THE $p$-ADIC NUMBERS
}

\author{
KEITH M. ROGERS
}

Let $\mathbb{Q}_{p}$ denote the $p$-adic numbers. We consider curves in $\mathbb{Q}_{p}^{n}$ defined by $p$-adic polynomials of one $p$-adic variable. We show that maximal averages along these curves are $L^{q}\left(\mathbb{Q}_{p}^{n}\right)$ bounded, where $1<q<\infty$.

\section{INTRODUCTION}

Let $P_{1}, \ldots, P_{n}$ be $p$-adic polynomials of one $p$-adic variable and define the curve $\gamma: \mathbb{Q}_{p} \longrightarrow \mathbb{Q}_{p}^{n}$ by $\gamma(t)=\left(P_{1}(t), \ldots, P_{n}(t)\right)$. We shall consider the averages

$$
\frac{1}{p^{k}} \int_{|t| \leqslant p^{k}} f(x-\gamma(t)) d t
$$

and the maximal average $\mathcal{M}_{\gamma} f(x)$ defined by

$$
\mathcal{M}_{\gamma} f(x)=\sup _{k \in \mathbf{Z}} \frac{1}{p^{k}} \int_{|t| \leqslant p^{k}} f(x-\gamma(t)) d t .
$$

We shall prove the following $p$-adic version of a theorem due to Stein and Wainger $[4,5]$.

THEOREM 1. Suppose that $1<q<\infty$, and that $\gamma$ and $\mathcal{M}_{\gamma}$ are defined as above. Then there is a constant $C_{q}$ so that

$$
\left\|\mathcal{M}_{\gamma} f\right\|_{L^{q}\left(\mathbb{Q}_{p}^{n}\right)} \leqslant C_{q}\|f\|_{L^{q}\left(\mathbb{Q}_{p}^{n}\right)},
$$

for all $f \in L^{q}\left(\mathbb{Q}_{p}^{n}\right)$.

The proof will mainly follow the Euclidean arguments. The difficulty lies in bounding oscillatory integrals on the $p$-adics, which was recently partially solved in [2].

We shall 'lift' to a higher dimensional situation and prove a similar theorem where the curve is defined by monomials. Then we 'descend' back to the original dimension and

Received 24th March, 2004

This is part of the author's Ph.D. thesis, written under the supervision of Professor Michael Cowling at the University of New South Wales. The author would like to thank Michael for all his invaluable help and also to Venta Terauds for proof reading this work.

Copyright Clearance Centre, Inc. Serial-fee code: 0004-9727/04 \$A2.00+0.00. 
curve. This technique goes back to de Leeuw [1]. In order to do this we let $R: \mathbb{Q}_{p}^{N} \rightarrow \mathbb{Q}_{p}^{n}$ be a linear map so that

$$
R\left(t, t^{2}, \ldots, t^{N}\right)=\left(P_{1}(t), \ldots P_{n}(t)\right)
$$

where $N$ is the maximal degree of the polynomials. We shall initially consider the curve $\widetilde{\gamma}(t)=\left(t, t^{2}, \ldots, t^{N}\right)$ and the maximal function

$$
\mathcal{M}_{\tilde{\gamma}} f(x)=\sup _{k \in \mathbf{Z}} \frac{1}{p^{k}} \int_{|t| \leqslant p^{k}} f(x-\widetilde{\gamma}(t)) d t .
$$

In Sections 2, 3 and 4 we shall introduce Fourier analysis on the $p$-adic numbers. In sections 5 and 6 we shall prove a version of the Hardy-Littlewood maximal theorem and obtain a Calderón-Zygmund type decomposition. In Sections 7 and 8 we shall prove $L^{2}\left(\mathbb{Q}_{p}^{N}\right)$ and $L^{q}\left(\mathbb{Q}_{p}^{N}\right)$ bounds for $\mathcal{M}_{\tilde{\gamma}}$. Finally, in Sections 9 and 10 , we shall finish the proof of Theorem 1 and consider the differentiation of integrals along curves. The constants $C$ take different values throughout.

\section{INTRODUCTION TO THE $p$-ADIC NUMBERS}

For a more complete introduction to the $p$-adic numbers, see [3] or [8]. Here we shall outline what we shall need.

Fix a prime number $p$. Any nonzero rational number $x$ can be uniquely expressed in the form $p^{k} m / n$, where $m$ and $n$ have no common divisors and neither is divisible by $p$. Define the $p$-adic norm on the rational numbers by $|x|=p^{-k}$ when $x \neq 0$, and $|0|=0$. We obtain the $p$-adic numbers by completing $\mathbb{Q}$ with respect to this norm. It is not difficult to show that the norm satisfies

$$
|x y|=|x||y|,
$$

and the ultrametric inequality,

$$
|x+y| \leqslant \max \{|x|,|y|\} .
$$

It follows from the ultrametric inequality, that every point within a ball can be considered to be its centre. Similarly, it can be shown that two balls are either disjoint or one is contained in the other.

A nonzero $p$-adic number $x$ such that $|x|=p^{-k}$ may be written in the form

$$
x=\sum_{j=k}^{\infty} x_{j} p^{j},
$$

where $0 \leqslant x_{j} \leqslant p-1$ and $x_{k} \neq 0$. This will be called the standard $p$-adic expansion and the arithmetic of these expansions is done formally, with carrying. 
As $\mathbb{Q}_{p}$ is a locally compact commutative group, there is a Haar measure, that necessarily satisfies $d(a x)=|a| d x$, where $d x$ denotes an element of this measure. We normalise so that $\left\{x \in \mathbb{Q}_{p}:|x| \leqslant p^{r}\right\}$ has measure $p^{r}$.

We shall be concerned with $n$-dimensional vector spaces over $\mathbb{Q}_{p}$. To this end let $\mathbb{Q}_{p}^{n}$ be the $n$-dimensional vector space over $\mathbb{Q}_{p}$, and let $|\cdot|$ denote the standard norm on $\mathbb{Q}_{p}^{n}$ defined by

$$
|x|=\max _{1 \leqslant j \leqslant n}\left|x_{j}\right|
$$

It is easy to show that this is also an ultrametric. A Haar measure is given by $d x=d x_{1} \ldots d x_{n}$, where $d x_{j}$ is the Haar measure on the $j$ th copy of $\mathbb{Q}_{p}$.

Balls defined using an ultrametric have some interesting properties. As

$$
\left\{y \in \mathbb{Q}_{p}^{n}:|y-x|<p^{r}\right\}=\left\{y \in \mathbb{Q}_{p}^{n}:|y-x| \leqslant p^{r-1}\right\},
$$

we see that they are both open and closed. Balls that contain the origin are subgroups. Every point within a ball can be considered to be its centre. Also, two balls are either disjoint or one is contained in the other.

We assume in general that all functions are complex-valued and Borel measurable. We define the compactly supported, locally constant functions to be the compactly supported functions that are constant on the cosets of some ball, and denote the space of these functions by $\mathcal{S}\left(\mathbb{Q}_{p}^{n}\right)$.

\section{FOURIER ANALYSIS ON THE $p$-ADIC NUMBERS}

In order to do Fourier analysis we shall need an understanding of the characters of $\mathbb{Q}_{p}$ and $\mathbb{Q}_{p}^{n}$. Define $\chi: \mathbb{Q}_{p} \longrightarrow \mathbb{C}$ by

$$
\chi(x)= \begin{cases}\prod_{j=k}^{-1} e^{2 \pi i x_{j} / p^{j}} & \text { when }|x|>1 \\ 1 & \text { otherwise. }\end{cases}
$$

The additive characters of $\mathbb{Q}_{p}$ are of the form $\chi_{a}: \mathbb{Q}_{p} \longrightarrow \mathbb{C}$;

$$
\chi_{a}(x)=\chi(a x),
$$

where $a \in \mathbb{Q}_{p}$, and the additive characters of $\mathbb{Q}_{p}^{n}$ are of the form $\chi_{a}: \mathbb{Q}_{p}^{n} \longrightarrow \mathbb{C}_{\text {; }}$

$$
\chi_{a}(x)=\chi(a \cdot x),
$$

where $a \in \mathbb{Q}_{p}^{n}$ and $a \cdot x=a_{1} x_{1}+\ldots+a_{n} x_{n}$.

The following result can be shown using the fact that balls have multiple centres. A proof can be found in [2] or [8]. 
Lemma 2. Suppose that $a \in \mathbb{Q}_{p}$ and $|a|>1$. Then

$$
\int_{|t| \leqslant 1} \chi(a t) d t=0
$$

The following van der Corput lemma for $p$-adic polynomials was recently proven in [2]. It will be key to the proof of Theorem 1.

Lemma 3. Suppose that $a_{1}, \ldots, a_{n} \in \mathbb{Q}_{p}$. Then

$$
\left|\int_{|t| \leqslant 1} \chi\left(a_{1} t+\cdots+a_{n} t^{n}\right) d t\right| \leqslant \frac{2 p^{n}}{\lambda^{1 / n}}
$$

where $\lambda=\max _{1 \leqslant j \leqslant n}\left|a_{j}\right|$.

The Fourier transform is defined by

$$
\widehat{f}(\xi)=\int_{\mathbb{Q}_{p}^{n}} f(x) \overline{\chi_{\xi}(x)} d x=\int_{\mathbb{Q}_{p}^{n}} f(x) \chi(-\xi \cdot x) d x
$$

for all $f \in L^{1}\left(\mathbb{Q}_{p}^{n}\right)$, and

$$
\widehat{\mu}(\xi)=\int_{\mathbb{Q}_{p}^{n}} \overline{\chi_{\xi}(x)} d \mu(x)=\int_{\mathbb{Q}_{p}^{n}} \chi(-\xi \cdot x) d \mu(x)
$$

for all finite Borel measures $\mu$. The Fourier transform maps functions in $\mathcal{S}\left(\mathbb{Q}_{p}^{n}\right)$ to functions in $\mathcal{S}\left(\mathbb{Q}_{p}^{n}\right)$, and $\mathcal{S}\left(\mathbb{Q}_{p}^{n}\right)$ is dense in $L^{q}\left(\mathbb{Q}_{p}^{n}\right)$, where $1 \leqslant q<\infty$. Thus, $\mathcal{S}\left(\mathbb{Q}_{p}^{n}\right)$ will take the role of the Schwartz function space.

Convolution is defined as usual by

$$
f * g(x)=\int_{\mathbb{Q}_{p}^{n}} f(x-y) g(y) d y=\int_{\mathbb{Q}_{p}^{n}} f(y) g(x-y) d y=g * f(x)
$$

and

$$
\mu * f(x)=\int_{\mathbb{Q}_{p}^{n}} f(x-y) d \mu(y)=f * \mu(x),
$$

for all functions $f, g$, and finite Borel measures $\mu$ for which the integral is defined. The following results follows as usual from the definitions and Fubini's theorem.

THEOREM 4. Suppose that $f, g \in L^{1}\left(\mathbb{Q}_{p}^{n}\right)$, and $\mu$ is a finite Borel measure. Then

$$
\|f * g\|_{L^{1}\left(\mathbb{Q}_{p}^{n}\right)} \leqslant\|f\|_{L^{1}\left(Q_{p}^{n}\right)}\|g\|_{L^{1}\left(\mathbb{Q}_{p}^{n}\right)}
$$

and

$$
\|f * \mu\|_{L^{1}\left(Q_{p}^{n}\right)} \leqslant\|f\|_{L^{1}\left(Q_{p}^{n}\right)}\|\mu\|_{M}
$$

where $\|\mu\|_{M}$ is the total variation of $\mu$. 
THEOREM 5. Suppose that $f, g \in L^{1}\left(\mathbb{Q}_{p}^{n}\right)$ and $\mu$ is a finite Borel measure. Then $\widehat{f * g}=\hat{f} \hat{g}$, and $\widehat{\mu * f}=\widehat{\mu} \widehat{f}$.

Finally, we present $p$-adic versions of the Fourier inversion theorem and Plancherel's theorem. For proofs see [8].

THEOREM 6. Suppose that $f \in L^{1}\left(\mathbb{Q}_{p}^{n}\right)$. Then for almost every $x \in \mathbb{Q}_{p}^{n}$,

$$
\int_{|y| \leqslant p^{k}} \widehat{f}(y) \chi_{x}(y) d y \rightarrow f(x)
$$

as $k \rightarrow \infty$.

THEOREM 7. Suppose that $f \in L^{1}\left(\mathbb{Q}_{p}^{n}\right) \cap L^{2}\left(\mathbb{Q}_{p}^{n}\right)$. Then $\|f\|_{L^{2}\left(\mathbb{Q}_{p}^{n}\right)}=\|\widehat{f}\|_{L^{2}\left(\mathbb{Q}_{p}^{n}\right)}$.

As $L^{1}\left(\mathbb{Q}_{p}^{n}\right) \cap L^{2}\left(\mathbb{Q}_{p}^{n}\right)$ is dense in $L^{2}\left(\mathbb{Q}_{p}^{n}\right)$ we can extend the definition of the Fourier transform to the whole of $L^{2}\left(\mathbb{Q}_{p}^{n}\right)$, as usual.

\section{Bessel potentials on the $p$-Adic NUMBERS}

It will be convenient to define $\Gamma_{n}$ by

$$
\Gamma_{n}(s)=\frac{1-p^{-n-s}}{1-p^{s}}
$$

where $s$ is a nonzero complex number. We shall require a $p$-adic analogue of the Bessel potential $\left(1+|x|^{2}\right)^{s / 2}$. Note that

$$
\left(1+|x|^{2}\right)^{s / 2}=\left(\left(1+|x|^{2}\right)^{1 / 2}\right)^{s}=\|(1, x)\|^{s},
$$

where $\|(1, x)\|$ is the $(n+1)$-dimensional Euclidean norm of $(1, x) \in \mathbb{R}^{n+1}$. Now the $(n+1)$-dimensional $p$-adic norm of $(1, x) \in \mathbb{Q}_{p}^{n+1}$ is $\max \{1,|x|\}$, so, following Taibleson $[7,8]$, we define the $n$-dimensional $p$-adic Bessel potential $J^{s}: \mathbb{Q}_{p}^{n} \longrightarrow \mathbb{C}$ by

$$
J^{s}(x)=\max \{1,|x|\}^{s},
$$

where $s \in \mathbb{C}$. We also define $K_{s}: \mathbb{Q}_{p}^{n} \longrightarrow \mathbb{C}$ by

$$
K_{s}(x)= \begin{cases}\left(|x|^{-n-s}-p^{-n-s}\right) / \Gamma_{n}(s) & \text { when }|x| \leqslant 1 \\ 0 & \text { otherwise, }\end{cases}
$$

when $s \neq-n$, and

$$
K_{-n}(x)= \begin{cases}\left(1-p^{-n}\right) \log _{p}(p /|x|) & \text { when }|x| \leqslant 1 \\ 0 & \text { otherwise. }\end{cases}
$$

It is easy to calculate that $K_{s} \in L^{1}\left(\mathbb{Q}_{p}^{n}\right)$ when $\operatorname{Re}(s)<0$. We shall require the following results due to Taibleson $[7,8]$. 
Proposition 8. Suppose that $\operatorname{Re}(s)<0$, and $J^{s}$ and $K_{s}$ are defined as above. Then $\widehat{J}^{s}(\xi)=K_{s}(\xi)$.

Lemma 9. Suppose that $J^{s}$ is defined as above and that $\operatorname{Re}(s)<0$. Then there is a constant $C_{s}$ so that

$$
\left\|\widehat{J}^{s}(\cdot+y)-\widehat{J}^{s}(\cdot)\right\|_{L^{1}\left(Q_{p}^{n}\right)} \leqslant C_{s}|y|^{-\operatorname{Re}(s)}
$$

for all $y \in \mathbb{Q}_{p}^{n}$.

\section{The Hardy-Littlewood maXimal theorem}

We shall make use of non-isotropic dilations $\rho_{k}: \mathbb{Q}_{p}^{N} \longrightarrow \mathbb{Q}_{p}^{N}$ defined by

$$
\rho_{k}(x)=\left(p^{k} x_{1}, p^{2 k} x_{2}, \ldots, p^{N k} x_{N}\right),
$$

and the norm $d: \mathbb{Q}_{p}^{N} \longrightarrow \mathbb{R}$ defined by

$$
d(x)=\max \left\{\left|x_{1}\right|,\left|x_{2}\right|^{1 / 2}, \ldots,\left|x_{N}\right|^{1 / N}\right\},
$$

in Sections 7 and 8. From now on balls will be defined using $d$, unless specified otherwise, so that

$$
B\left(x, p^{k}\right)=\left\{y: d(x-y) \leqslant p^{k}\right\}=\left\{y:\left|y_{j}-x_{j}\right| \leqslant p^{j k} \text { for all } j=1, \ldots, N\right\},
$$

and $\left|B\left(x, p^{k}\right)\right|=p^{M k}$, where $M=1+2+\ldots+N=N(N+1) / 2$. Balls denoted by $B^{j}$ for some $j \in \mathbb{N}$, will also be defined using $d$, but will have no specified radius or position. It is not difficult to show that $d$ is an ultrametric. Thus, all points within a ball can be considered to be its centre, and two balls are disjoint or one is contained in the other, as before.

We begin with a covering lemma in the style of $\mathrm{N}$. Wiener. We note that the technicality is significantly reduced when dealing with ultrametrics.

Lemma 10. Suppose that $E \subset \mathbb{Q}_{p}^{N}$ has finite Haar measure, and is covered by balls defined with an ultrametric. Suppose that the balls have uniformly bounded measure. Then there exists a countable and disjoint subcover.

Proof: We choose a refinement from the original cover. We start with a ball with largest measure, and discard all the balls contained within it. Then we choose a ball with largest measure from the remaining balls, and discard the balls which are contained within it. We continue until all the balls have been chosen or discarded.

The refinement is disjoint as any two balls are disjoint or one is contained in the other. It is countable as $E$ is of finite measure, and each ball has positive measure. Finally it is a cover, as we only discarded redundant elements of the original cover. 
We shall now define the Hardy-Littlewood maximal function $\mathcal{M} f$ for a function $f \in L_{\text {loc }}^{1}\left(\mathbb{Q}_{p}^{N}\right)$. Define $\Phi_{k}$ by

$$
\Phi_{k}=\frac{1}{\left|B\left(0, p^{k}\right)\right|} 1_{B\left(0, p^{k}\right)}
$$

where $1_{B\left(0, p^{k}\right)}$ denotes the characteristic function of $B\left(0, p^{k}\right)$, and $\mathcal{M} f$ by

$$
\mathcal{M} f(x)=\sup _{k \in \mathbf{Z}} \frac{1}{\left|B\left(x, p^{k}\right)\right|} \int_{B\left(x, p^{k}\right)}|f(y)| d y=\sup _{k \in \mathbf{Z}} \Phi_{k} *|f| .
$$

As all points within a ball can be considered to be its centre, this is the analogue of both the centred and uncentred maximal function of the classical theory.

Theorem 11. Suppose that $\mathcal{M} f$ is defined as above. Then

$$
\left|\left\{x \in \mathbb{Q}_{p}^{N}: \mathcal{M} f(x)>\alpha\right\}\right| \leqslant \frac{1}{\alpha}\|f\|_{L^{1}\left(\mathbb{Q}_{p}^{N}\right)},
$$

for all $f \in L^{1}\left(\mathbb{Q}_{p}^{N}\right)$, and for $q>1$,

$$
\|\mathcal{M} f\|_{L^{q}\left(\mathbb{Q}_{p}^{N}\right)} \leqslant 2\left(\frac{q}{q-1}\right)^{1 / q}\|f\|_{L^{q}\left(\mathbb{Q}_{p}^{N}\right)}
$$

for all $f \in L^{q}\left(\mathbb{Q}_{p}^{N}\right)$.

PROOF: Let $F$ be any subset of $\left\{x \in \mathbb{Q}_{p}^{N}: \mathcal{M} f(x)>\alpha\right\}$ with finite measure. For each $x \in F$ there exists a ball $B(x)$ so that

$$
|B(x)|<\frac{1}{\alpha} \int_{B(x)}|f(x)| d x \leqslant \frac{1}{\alpha}\|f\|_{L^{1}} .
$$

We can apply Lemma 10 to the cover $\{B(x)\}_{x \in F}$, to leave a countable, disjoint subcover $\left\{B^{j}\right\}_{j \in \mathbf{N}}$. Thus

$$
|F| \leqslant \sum_{j=0}^{\infty}\left|B^{j}\right|<\sum_{j=0}^{\infty} \frac{1}{\alpha} \int_{B^{j}}|f(x)| d x \leqslant \frac{1}{\alpha} \int_{\mathbb{Q}_{p}^{N}}|f(x)| d x .
$$

Now this is true for all subsets of $\left\{x \in \mathbb{Q}_{p}^{N}: \mathcal{M} f(x)>\alpha\right\}$ with finite measure, so that

$$
\left|\left\{x \in \mathbb{Q}_{p}^{N}: \mathcal{M} f(x)>\alpha\right\}\right| \leqslant \frac{1}{\alpha} \int_{\mathbb{Q}_{p}^{N}}|f(x)| d x,
$$

as desired.

To prove the second part, we split $f$ as $f^{\alpha}+f_{\alpha}$, where

$$
f^{a}(x)= \begin{cases}f(x) & \text { when }|f(x)|>\alpha / 2 \\ 0 & \text { otherwise }\end{cases}
$$


and

$$
f_{\alpha}(x)= \begin{cases}f(x) & \text { when }|f(x)| \leqslant \alpha / 2 \\ 0 & \text { otherwise. }\end{cases}
$$

As $\left\{x \in \mathbb{Q}_{p}^{N}: \mathcal{M} f_{\alpha}>\alpha / 2\right\}=\emptyset$, we see that

$$
\{\mathcal{M} f>\alpha\} \subseteq\left\{\mathcal{M} f^{\alpha}>\alpha / 2\right\} \cup\left\{\mathcal{M} f_{\alpha}>\alpha / 2\right\}=\left\{\mathcal{M} f^{\alpha}>\alpha / 2\right\},
$$

where $\{\mathcal{M} f>\alpha\}$ denotes $\left\{x \in \mathbb{Q}_{p}^{N}: \mathcal{M} f(x)>\alpha\right\}$ as usual. By (4) we have

$$
\left|\left\{\mathcal{M} f^{\alpha}>\alpha / 2\right\}\right| \leqslant \frac{2}{\alpha} \int_{\mathbb{Q}_{p}^{N}}\left|f^{\alpha}(x)\right| d x=\frac{2}{\alpha} \int_{\{|f|>\alpha / 2\}}|f(x)| d x,
$$

so that

$$
\begin{aligned}
\int_{\mathbb{Q}_{p}^{N}}|\mathcal{M} f(x)|^{q} d x & =\int_{0}^{\infty} q \alpha^{q-1}|\{\mathcal{M} f>\alpha\}| d \alpha \\
& \leqslant 2 q \int_{0}^{\infty} \alpha^{q-2} \int_{\{|f|>\alpha / 2\}}|f(x)| d x d \alpha .
\end{aligned}
$$

Thus, by changing the order of integration, we obtain

$$
\int_{\mathbb{Q}_{p}^{N}}|\mathcal{M} f(x)|^{q} d x \leqslant 2 q \int_{\mathbb{Q}_{p}^{N}}|f(x)| \int_{0}^{2|f|} \alpha^{q-2} d \alpha d x=\frac{2^{q} q}{q-1} \int_{\mathbb{Q}_{P}^{N}}|f(x)|^{q} d x,
$$

as desired.

The bound in (3) is absolutely sharp, and this is easily observed by considering $f=1_{B(0,1)}$.

An important corollary of the Hardy-Littlewood maximal theorem is the following differentiation theorem.

CoRollarY 12. Suppose that $f \in L^{1}\left(\mathbb{Q}_{p}^{N}\right)$. Then for almost every $x \in \mathbb{Q}_{p}^{N}$,

$$
\lim _{k \rightarrow-\infty} \frac{1}{\left|B\left(x, p^{k}\right)\right|} \int_{B\left(x, p^{k}\right)} f(y) d y=f(x) .
$$

Proof: It will suffice to show that $\left|E_{\delta}\right|=0$ for all $\delta>0$, where

$$
E_{\delta}=\left\{x \in \mathbb{Q}_{p}^{N}: \limsup _{k \rightarrow-\infty}\left|\frac{1}{\left|B\left(x, p^{k}\right)\right|} \int_{B\left(x, p^{k}\right)} f(y) d y-f(x)\right|>\delta\right\} .
$$

Let $\varepsilon>0$ and $g_{\varepsilon} \in \mathcal{S}\left(\mathbb{Q}_{p}^{N}\right)$ such that $\left\|f-g_{\varepsilon}\right\|_{L^{1}} \leqslant \varepsilon$. Now when $p^{k}$ is sufficiently small,

$$
\frac{1}{\left|B\left(x, p^{k}\right)\right|} \int_{B\left(x, p^{k}\right)} g_{\varepsilon}(y) d y=g_{\varepsilon}(x)
$$


for all $x \in \mathbb{Q}_{p}^{N}$, so that

$$
\frac{1}{\left|B\left(x, p^{k}\right)\right|} \int_{B\left(x, p^{k}\right)} f(y) d y-f(x)=\frac{1}{\left|B\left(x, p^{k}\right)\right|} \int_{B\left(x, p^{k}\right)}\left(f(y)-g_{\varepsilon}(y)\right) d y+g_{\varepsilon}(x)-f(x) .
$$

Thus, $E_{\delta} \subset A_{\delta} \cup B_{\delta}$, where

$$
A_{\delta}=\left\{x \in \mathbb{Q}_{p}^{N}:\left|\limsup _{k \rightarrow-\infty} \frac{1}{\left|B\left(x, p^{k}\right)\right|} \int_{B\left(x, p^{k}\right)}\left(f(y)-g_{\varepsilon}(y)\right) d y\right|>\delta / 2\right\}
$$

and

$$
B_{\delta}=\left\{x \in \mathbb{Q}_{p}^{N}:\left|g_{\varepsilon}(x)-f(x)\right|>\delta / 2\right\}
$$

Now by Theorem 11,

$$
\left|\left\{x \in \mathbb{Q}_{p}^{N}:\left|\limsup _{k \rightarrow-\infty} \frac{1}{\left|B\left(x, p^{k}\right)\right|} \int_{B\left(x, p^{k}\right)}\left(f(y)-g_{\varepsilon}(y)\right) d y\right|>\delta / 2\right\}\right| \leqslant \frac{2}{\delta}\left\|f-g_{\varepsilon}\right\|_{L^{1}},
$$

and by Chebyshev's inequality,

$$
\left|\left\{x \in \mathbb{Q}_{p}^{N}:\left|g_{\varepsilon}(x)-f(x)\right|>\delta / 2\right\}\right| \leqslant \frac{2}{\delta}\left\|f-g_{\varepsilon}\right\|_{L^{1}},
$$

so that

$$
\left|E_{\delta}\right| \leqslant \frac{2}{\delta}\left\|f-g_{\varepsilon}\right\|_{L^{1}}+\frac{2}{\delta}\left\|f-g_{\varepsilon}\right\|_{L^{1}}=\frac{4 \varepsilon}{\delta} .
$$

Finally we let $\varepsilon$ tend to zero to see that $\left|E_{\delta}\right|=0$.

\section{The Calderón-Zygmund decomposition}

The following proposition splits an integrable function into a large and a small part. The small part will naturally be easy to bound, and the large part will have some redeeming qualities.

Proposition 13. Suppose that $f \in L^{1}\left(\mathbb{Q}_{p}^{N}\right)$ and that $\alpha>0$. Then

$$
f=g+\sum_{j=0}^{\infty} b_{j}
$$

where $g, b_{j} \in L^{1}\left(\mathbb{Q}_{p}^{N}\right)$ and each $b_{j}$ is supported in a ball $B^{j}$, so that

(i) $|g(x)| \leqslant \alpha$ for almost every $x$,

(ii) $\left\|b_{j}\right\|_{L^{1}\left(\mathbb{Q}_{p}^{N}\right)} \leqslant 2 \alpha\left|B^{j}\right|$,

(iii) $\int_{\mathbb{Q}_{p}^{N}} b_{j}(x) d x=0$,

(iv) $\sum_{j}\left|B^{j}\right| \leqslant \frac{p^{M}}{\alpha}\|f\|_{L^{1}\left(Q_{p}^{N}\right)}$. 
Proof: Define $E=\left\{x \in \mathbb{Q}_{p}^{N}: \mathcal{M} f(x)>\alpha\right\}$, where $\mathcal{M} f$ is the Hardy-Littlewood maximal function, defined with our new balls and norm. For $x \in \mathbb{Q}_{p}^{N}$ let $\delta(x)$ be the minimal distance such that

$$
B(x, \delta(x)) \cap E^{c} \neq \emptyset .
$$

The set $E$ is of finite measure, by Theorem 11 , and as $B(x, \delta(x) / p) \subset E$ we see that the balls are of uniformly bounded measure. Thus we can apply Lemma 10 to $\{B(x, \delta(x))\}_{x \in E}$, to obtain a countable and disjoint subcover of $E$, which we denote by $\left\{B^{j}\right\}$.

Define $g$ by

$$
g(x)=\left\{\begin{array}{lll}
f(x) & \text { when } & x \notin E \\
\frac{1}{\left|B^{j}\right|} \int_{B^{j}} f(y) d y & \text { when } & x \in B_{j}
\end{array}\right.
$$

and $b_{j}$ by

$$
b_{j}(x)=1_{B^{j}}(x)\left(f(x)-\frac{1}{\left|B^{j}\right|} \int_{B^{j}} f(y) d y\right)
$$

so that $f=g+\sum_{j} b_{j}$.

When $x \notin E$, we see by Corollary 12 that $g(x) \leqslant \alpha$ for almost every $x$. When $x \in E$,

$$
g(x)=\frac{1}{\left|B^{j}\right|} \int_{B^{j}} f(y) d y \leqslant \alpha,
$$

as $B^{j} \cap E^{c} \neq \emptyset$, so we have proven the first part. Similarly as $B^{j} \cap E^{c} \neq \emptyset$,

$$
\begin{aligned}
\int_{\mathbb{Q}_{p}^{N}}\left|b_{j}(x)\right| d x & \leqslant 2 \int_{B^{j}}|f(x)| d x \\
& =2\left|B^{j}\right| \frac{1}{\left|B^{j}\right|} \int_{B^{j}}|f(x)| d x \leqslant 2\left|B^{j}\right| \alpha,
\end{aligned}
$$

so the second part holds. The third part is clear by the definition of $b_{j}$. Finally we consider the balls $B_{*}^{j}$, where $B\left(x, p^{k}\right)_{*}=B\left(x, p^{k-1}\right)$. It is clear that the $B_{*}^{j}$ are disjoint, and that $\cup B_{*}^{j} \subset E$. Thus

$$
\sum_{j}\left|B^{j}\right|=p^{M} \sum_{j}\left|B_{*}^{j}\right| \leqslant p^{M}|E| \leqslant \frac{p^{M}}{\alpha}\|f\|_{L^{1}}
$$

by Theorem 11, and we are done.

\section{7. $L^{2}$ BOUNDEDNESS}

It will be useful to consider $\mathcal{M}_{\tilde{\gamma}}$ as a maximal convolution of measures. To this end we let $\mu$ be the measure defined by

$$
\int_{\mathbf{Q}_{p}^{N}} f(x) d \mu(x)=\int_{|t| \leqslant 1} f(\widetilde{\gamma}(t)) d t
$$


where $\tilde{\gamma}(t)=\left(t, t^{2}, \ldots, t^{N}\right)$, and let $\mu_{k}$ be the measure defined by

$$
\int_{\mathbb{Q}_{p}^{N}} f(x) d \mu_{k}(x)=\int_{\mathbb{Q}_{p}^{N}} f(x) d \mu\left(\rho_{k}(x)\right)=\frac{1}{p^{k}} \int_{|t| \leqslant p^{k}} f(\tilde{\gamma}(t)) d t .
$$

Then $\mathcal{M}_{\tilde{\gamma}} f$ is defined by

$$
\mathcal{M}_{\tilde{\gamma}} f(x)=\sup _{\boldsymbol{k} \in \mathbf{Z}} \frac{1}{p^{k}} \int_{|t| \leqslant p^{k}} f(x-\tilde{\gamma}(t)) d t=\sup _{k \in \mathbf{Z}} \mu_{k} * f .
$$

The use of square functions will be key to the proof of the following proposition. This idea was developed by Stein and Wainger $[\mathbf{4}, \mathbf{5}]$.

Proposition 14. Suppose that $\mathcal{M}_{\tilde{\gamma}}$ is defined as above. Then there is a constant $C_{N}$ so that

$$
\left\|\mathcal{M}_{\tilde{\gamma}} f\right\|_{L^{2}\left(\mathbb{Q}_{p}^{N}\right)} \leqslant C_{N}\|f\|_{L^{2}\left(\mathbb{Q}_{p}^{N}\right)}
$$

for all $f \in \mathcal{S}\left(\mathbb{Q}_{p}^{N}\right)$.

Proof: Define the square function $\mathcal{G} f$ by

$$
\mathcal{G} f(x)=\left(\sum_{k \in \mathbf{Z}}\left|\mu_{k} * f-\Phi_{k} * f\right|^{2}\right)^{1 / 2},
$$

where

$$
\Phi_{k}(\xi)=\frac{1}{\left|B\left(0, p^{k}\right)\right|} 1_{B\left(0, p^{k}\right)}
$$

as before, so that

$$
\mathcal{G} f(x)=\left(\sum_{k \in \mathbf{Z}}\left|\frac{1}{p^{k}} \int_{|t| \leqslant p^{k}}\right| f(x-\tilde{\gamma}(t))\left|d t-\frac{1}{p^{M k}} \int_{B\left(x, p^{k}\right)}\right| f(x-s)|d s|^{2}\right)^{1 / 2},
$$

where $M=N(N+1) / 2$. We note that $x$ and $s$ are vector-valued, and $t$ is scalar-valued. Now

$$
\mathcal{M}_{\tilde{\gamma}} f(x) \leqslant \mathcal{G} f(x)+\mathcal{M} f(x)
$$

and $\mathcal{M}$ is bounded by Theorem 11. Thus, it remains to show that $\mathcal{G}$ is bounded. If we assume for the moment that $\mathcal{G} f \in L^{2}\left(\mathbb{Q}_{p}^{N}\right)$, then

$$
\|\mathcal{G} f\|_{L^{2}}^{2}=\sum_{k \in \mathbf{Z}}\left\|\mu_{k} * f-\Phi_{k} * f\right\|_{L^{2}}^{2},
$$

so that by Plancherel's theorem,

$$
\|\mathcal{G} f\|_{L^{2}}^{2}=\int_{\mathbf{Q}_{p}^{N}}|\widehat{f}(\xi)|^{2} \sum_{k \in \mathbf{Z}}\left|m_{k}(\xi)\right|^{2} d \xi
$$


where $m_{k}(\xi)=\widehat{\mu_{k}}(\xi)-\widehat{\Phi_{k}}(\xi)$. Thus

$$
m_{k}(\xi)=\int_{|t| \leqslant 1} \chi\left(\frac{\xi_{1}}{p^{k}} t+\frac{\xi_{2}}{p^{2 k}} t^{2}+\cdots+\frac{\xi_{N}}{p^{N k}} t^{N}\right) d t-\prod_{j=1}^{N} \int_{\left|t_{j}\right| \leqslant 1} \chi\left(\frac{\xi_{j}}{p^{j k}} t_{j}\right) d t_{j}
$$

When $\left|\xi_{j}\right| p^{j k} \leqslant 1$ for all $j$, we have

$$
\int_{|t| \leqslant 1} \chi\left(\frac{\xi_{1}}{p^{k}} t+\frac{\xi_{2}}{p^{2 k}} t^{2}+\cdots+\frac{\xi_{N}}{p^{N k}} t^{N}\right) d t=1
$$

as $\chi$ is trivial on $\left\{x \in \mathbb{Q}_{p}:|x| \leqslant 1\right\}$. Similarly

$$
\prod_{j=1}^{N} \int_{\left|t_{j}\right| \leqslant 1} \chi\left(\frac{\xi_{j}}{p^{j k}} t_{j}\right) d t_{j}=1,
$$

so that $m_{k}(\xi)=0$. When $\left|\xi_{j}\right| p^{j k}>1$ for some $j$, we have

$$
\prod_{j=1}^{N} \int_{\left|t_{j}\right| \leqslant 1} \chi\left(\frac{\xi_{j}}{p^{j k}} t_{j}\right) d t_{j}=0
$$

by Lemma 2 , so that

$$
\left|m_{k}(\xi)\right|=\left|\int_{|t| \leqslant 1} \chi\left(\frac{\xi_{1}}{p^{k}} t+\cdots+\frac{\xi_{N}}{p^{N k}} t^{N}\right) d t\right| \leqslant \frac{p^{N}}{\max _{j}\left\{\left|\xi_{j}\right| p^{j k}\right\}^{1 / N}},
$$

by Lemma 3 . Thus

$$
\sum_{k \in \mathbb{Z}}\left|m_{k}(\xi)\right|^{2} \leqslant \sum_{\substack{k \in \mathbb{Z} \\ \max _{j}\left\{\left|\xi_{j}\right| p^{j k}\right\}>1}}\left(\frac{p^{N}}{\max _{j}\left\{\left|\xi_{j}\right| p^{j k}\right\}^{1 / N}}\right)^{2} \leqslant C_{N}
$$

for some constant $C_{N}$. Thus

$$
\|\mathcal{G} f\|_{L^{2}}^{2}=\int_{\mathbb{Q}_{P}^{N}}|\widehat{f}(\xi)|^{2} \sum_{k \in \mathbf{Z}}\left|m_{k}(\xi)\right|^{2} d \xi \leqslant C_{N}\|\widehat{f}\|_{L^{2}}^{2}=C_{N}\|f\|_{L^{2}}^{2},
$$

by Plancherel's theorem, and we are done.

In order to take advantage of the 'slack' in the above argument we introduce the measures $\nu^{s}$ defined by

$$
\widehat{\nu^{s}}(x)=\widehat{\mu}(x) \max \{1,|x|\}^{s}
$$

and $\nu_{k}^{s}$ defined by

$$
\widehat{\nu_{k}^{s}}(x)=\widehat{\nu^{s}}\left(\rho_{-k}(x)\right)=\widehat{\mu}\left(\rho_{-k}(x)\right) \max \left\{1,\left|\rho_{-k}(x)\right|\right\}^{s},
$$


where $s \in \mathbb{C}$. By a change of variables,

$$
\widehat{\nu_{k}^{s}}(x)=\widehat{\mu_{k}}(x) \max \left\{1,\left|\rho_{-k}(x)\right|\right\}^{s}
$$

so by the proof of Proposition 14, we see that $\mathcal{N}_{s}$ defined by

$$
\mathcal{N}_{s} f=\sup _{k \in \mathbf{Z}} \nu_{k}^{s} * f
$$

is also $L^{2}\left(\mathbb{Q}_{p}^{N}\right)$ bounded when $\operatorname{Re}(s)<1 / N$. We state this formally for future reference.

Proposition 15. Suppose $\mathcal{N}_{s}$ is defined as above. Then for each $s \in \mathbb{C}$ such that $\operatorname{Re}(s)<1 / N$, there is a constant $C_{s}$ so that

$$
\left\|\mathcal{N}_{s} f\right\|_{L^{2}\left(\mathbb{Q}_{p}^{N}\right)} \leqslant C_{s}\|f\|_{L^{2}\left(\mathbb{Q}_{p}^{N}\right)}
$$

for all $f \in \mathcal{S}\left(\mathbb{Q}_{p}^{N}\right)$.

\section{8. $L^{q}$ BOUNDEDNESS}

We aim to bound $\mathcal{N}_{s}$ when $\operatorname{Re}(s)<0$. This will enable us to use complex interpolation to obtain a bound for $\mathcal{M}_{\tilde{\gamma}}$.

From (5) we can calculate, using Theorem 5, that

$$
\nu_{k}^{s}(x)=\frac{1}{p^{M k}} \mu * \widehat{J}^{s}\left(\rho_{k}(x)\right),
$$

where $J^{s}$ is the Bessel potential defined as in (2). Thus $\nu_{k}^{s}$ is an $L^{1}\left(\mathbb{Q}_{p}^{N}\right)$ function when $\operatorname{Re}(s)<0$, by Proposition 8 and Theorem 4 , and by a change in variables $\left\|\nu_{k}^{s}\right\|_{L^{1}} \leqslant\left\|\widehat{J}^{s}\right\|_{L^{1}}$ for all $k \in \mathbb{Z}$.

One of the reasons we have been considering the norm $d$ is so that we can obtain the following version of Hörmander's condition. When $d(x)>d(y)$, we have

$$
d\left(\rho_{k}(x)\right)>d\left(\rho_{k}(y)\right)
$$

for all $k \in \mathbb{Z}$. It is easy to see that $|x| \geqslant d(x)$ when $d(x) \geqslant 1$. We also note that as $d$ is an ultrametric, $d(x+y)=d(x)$ when $d(x)>d(y)$.

Proposition 16. Suppose that $\nu_{k}^{s}$ is defined as above, and that $\operatorname{Re}(s)<0$. Then there is a constant $C_{s}$ so that

$$
\int_{d(x)>d(y)} \sup _{k}\left|\nu_{k}^{s}(x-y)-\nu_{k}^{s}(x)\right| d x \leqslant C_{s}
$$

for all $y \in \mathbb{Q}_{p}^{N}$. 
Proof: First we note that

$$
\begin{aligned}
I & =\int_{d(x)>d(y)} \sup _{k}\left|\nu_{k}^{s}(x-y)-\nu_{k}^{s}(x)\right| d x \\
& \leqslant \sum_{k} \int_{d(x)>d(y)}\left|\nu_{k}^{s}(x-y)-\nu_{k}^{s}(x)\right| d x \\
& =\sum_{k} \int_{d(x)>d(y)} \frac{1}{p^{M k}}\left|\int_{|t| \leqslant 1} \widehat{J}^{s}\left(\rho_{k}(x-y)-\widetilde{\gamma}(t)\right)-\widehat{J}^{s}\left(\rho_{k}(x)-\tilde{\gamma}(t)\right) d t\right| d x .
\end{aligned}
$$

When $\left|\rho_{k}(y)\right| \geqslant 1$, we have $d\left(\rho_{k}(y)\right) \geqslant 1$, so that

$$
\left|\rho_{k}(x-y)\right| \geqslant d\left(\rho_{k}(x-y)\right)>d\left(\rho_{k}(y)\right) \geqslant 1,
$$

as $d(x-y)=d(x)>d(y)$. Hence, as $\widehat{J}^{s}$ is supported in the unit ball,

$$
\widehat{J}^{s}\left(\rho_{k}(x-y)-\widetilde{\gamma}(t)\right)=0
$$

for all $|t| \leqslant 1$. Similarly when $\left|\rho_{k}(y)\right| \geqslant 1$,

$$
\left|\rho_{k}(x)\right| \geqslant d\left(\rho_{k}(x)\right)>d\left(\rho_{k}(y)\right) \geqslant 1,
$$

so that

$$
\widehat{J}^{s}\left(\rho_{k}(x)-\tilde{\gamma}(t)\right)=0
$$

for all $|t| \leqslant 1$. Thus, by Fubini's theorem,

$$
I \leqslant \sum_{k:\left|\rho_{k}(y)\right|<1} \int_{|t| \leqslant 1} \frac{1}{p^{M k}} \int_{\mathbb{Q}_{p}^{N}}\left|\widehat{J}^{s}\left(\rho_{k}(x-y)-\widetilde{\gamma}(t)\right)-\widehat{J}^{s}\left(\rho_{k}(x)-\widetilde{\gamma}(t)\right)\right| d x d t,
$$

so by the change of variables $z=\rho_{k}(x)-\tilde{\gamma}(t)$,

$$
I \leqslant \sum_{k:\left|\rho_{k}(y)\right|<1} \int_{|t| \leqslant 1} \int_{\mathbb{Q}_{p}^{N}}\left|\widehat{J}^{s}\left(z-\rho_{k}(y)\right)-\widehat{J}^{s}(z)\right| d z d t
$$

Finally, by Lemma 9 ,

$$
I \leqslant \sum_{k:\left|\rho_{k}(y)\right|<1} \int_{|t| \leqslant 1} C_{s}\left|\rho_{k}(y)\right|^{-\operatorname{Re}(s)} d t=C_{s} \sum_{k:\left|\rho_{k}(y)\right|<1}\left|\rho_{k}(y)\right|^{-\operatorname{Re}(s)} \leqslant C_{s}^{\prime},
$$

as desired.

We use this and our Calderón-Zygmund decomposition to prove the following proposition.

Propos Ition 17. Suppose that $1<q \leqslant \infty$ and that $\mathcal{N}^{s}$ is defined as above. Then for each $s \in \mathbb{C}$ such that $\operatorname{Re}(s)<0$, there is a constant $C_{q, s}$ so that

$$
\left\|\mathcal{N}_{s} f\right\|_{L^{q}\left(\mathbf{Q}_{p}^{N}\right)} \leqslant C_{q, s}\|f\|_{L^{q}\left(\mathbf{Q}_{p}^{N}\right)}
$$

for all $f \in \mathcal{S}\left(\mathbb{Q}_{p}^{N}\right)$. 
Proof: When $\operatorname{Re}(s)<0$, we have

$$
\sup _{x \in \mathbb{Q}_{p}^{N}}\left|\nu_{k}^{s} * f(x)\right| \leqslant\left\|\nu_{k}^{s}\right\|_{L^{1}}\|f\|_{L^{\infty}} \leqslant\left\|\widehat{J}^{s}\right\|_{L^{1}}\|f\|_{L^{\infty}}
$$

for all $k \in \mathbb{Z}$. Hence there is a constant $A_{s}=\left\|\widehat{J}^{s}\right\|_{L^{1}}$ so that

$$
\sup _{x \in \mathbb{Q}_{p}^{N}}\left|\mathcal{N}_{s} f(x)\right| \leqslant A_{s}\|f\|_{L^{\infty}}
$$

for all $f \in \mathcal{S}\left(\mathbb{Q}_{p}^{N}\right)$. We shall show that $\mathcal{N}_{s}$ is weak type $(1,1)$ and then interpolate using (6) to obtain the result.

We split $f$ into two parts $g$ and $b=\sum_{j} b_{j}$ as in Proposition 13 , so that

$$
\left\{x \in \mathbb{Q}_{p}^{N}: \mathcal{N}_{s} g(x)>A_{s} \alpha\right\}=\emptyset
$$

As

$$
\left\{x: \mathcal{N}_{s} f(x)>2 A_{s} \alpha\right\} \subset\left\{x: \mathcal{N}_{s} g(x)>A_{s} \alpha\right\} \cup\left\{x: \mathcal{N}_{s} b(x)>A_{s} \alpha\right\},
$$

it remains to bound $\left|\left\{x \in \mathbb{Q}_{p}^{N}: \mathcal{N}_{s} b(x)>A_{s} \alpha\right\}\right|$. Let $b_{j}$ be supported on $B^{j}=B\left(x_{j}, \delta_{j}\right)$, say, then

$$
\begin{aligned}
I & =\int_{\left(\cup B^{j}\right)^{c}}\left|\mathcal{N}_{s} b(x)\right| d x \leqslant \int_{\left(\cup B^{j}\right)^{c}} \sup _{k}\left|\nu_{k}^{s} * b(x)\right| d x \\
& =\int_{\left(\cup B^{j}\right)^{c}} \sup _{k}\left|\sum_{j} \int_{B^{j}} b_{j}(y) \nu_{k}^{s}(x-y) d y\right| d x .
\end{aligned}
$$

By (iii) in Proposition 13, we have $\int_{B^{j}} b_{j}(y) \nu_{k}^{s}\left(x-x_{j}\right) d y=0$, so that

$$
\begin{aligned}
I & \leqslant \int_{\left(\cup B^{j}\right)^{c}} \sup _{k}\left|\sum_{j} \int_{B^{j}} b_{j}(y)\left(\nu_{k}^{s}(x-y)-\nu_{k}^{s}\left(x-x_{j}\right)\right) d y\right| d x \\
& \leqslant \sum_{j} \int_{\left(B^{j}\right)^{c}} \sup _{k}\left|\nu_{k}^{s}(x-y)-\nu_{k}^{s}\left(x-x_{j}\right)\right| d x \int_{B^{j}}\left|b_{j}(y)\right| d y .
\end{aligned}
$$

Now if we make the change of variables $z=x-x_{j}$, then we see that

$$
I \leqslant \sum_{j} \int_{B\left(0, \delta_{j}\right)^{c}} \sup _{k}\left|\nu_{k}^{s}\left(z+x_{j}-y\right)-\nu_{k}^{s}(z)\right| d z \int_{B^{j}}\left|b_{j}(y)\right| d y .
$$

Now $d(z)>\delta_{j}$, as $x \in B\left(x_{j}, \delta_{j}\right)^{c}$, and $d\left(y-x_{j}\right) \leqslant \delta_{j}$, as $y \in B\left(x_{j}, \delta_{j}\right)$. Thus

$$
\int_{B\left(0, \delta_{j}\right)^{c}} \sup _{k}\left|\nu_{k}^{s}\left(z-\left(y-x_{j}\right)\right)-\nu_{k}^{s}(z)\right| d z \leqslant C_{s}
$$

by Proposition 16 , so that

$$
I \leqslant \sum_{j} C_{s} \int_{B^{j}}\left|b_{j}(y)\right| d y
$$


By (ii) and (iv) of Proposition 16,

$$
I=\int_{\left(U B^{j}\right)^{c}}\left|\mathcal{N}_{s} b(x)\right| d x \leqslant C_{s} \sum_{j} 2 \alpha\left|B^{j}\right| \leqslant 2 C_{s} p^{M}\|f\|_{L^{1}}
$$

so that

$$
\left|\left\{x \in \mathbb{Q}_{p}^{N}: \mathcal{N}_{s} b(x)>A_{s} \alpha\right\}\right| \leqslant \frac{2 C_{s} p^{M}}{A_{s} \alpha}\|f\|_{L^{1}}+\left|\cup B^{j}\right| .
$$

Thus by (iv) in Proposition 16 again, we see that

$$
\left|\left\{x \in \mathbb{Q}_{p}^{N}: \mathcal{N}_{s} b(x)>A_{s} \alpha\right\}\right| \leqslant \frac{\left(2 C_{s} / A_{s}+1\right) p^{M}}{\alpha}\|f\|_{L^{1}} .
$$

Finally, as $\left\{x \in \mathbb{Q}_{p}^{N}: \mathcal{N}_{s} f(x)>2 A_{s} \alpha\right\} \subset\left\{x \in \mathbb{Q}_{p}^{N}: \mathcal{N}_{s} b(x)>A_{s} \alpha\right\}$,

$$
\left|\left\{x \in \mathbb{Q}_{p}^{N}: \mathcal{N}_{s} f(x)>\alpha\right\}\right| \leqslant \frac{2\left(2 C_{s}+A_{s}\right) p^{M}}{\alpha}\|f\|_{L^{1}}
$$

We interpolate between this bound and the bound in (6) using the argument in the proof of Theorem 11, and we are done.

Finally we require the following complex interpolation theorem due to Stein [6]. We reformulate it in the generality that we require. Let

$$
D=\{z \in \mathbb{C}: a \leqslant \operatorname{Re}(z) \leqslant b\},
$$

and call a family of operators $\left\{\mathcal{T}_{z}\right\}_{z \in \mathbb{C}}$ admissible if, for $f, g \in \mathcal{S}\left(\mathbb{Q}_{p}^{N}\right)$, the mapping

$$
z \mapsto \int_{\mathbb{Q}_{p}^{N}}\left(\mathcal{T}_{z} f(x)\right) g(x) d x
$$

is analytic in the interior of $D$, continuous on $D$, and uniformly bounded on $D$.

TheOREM 18. Suppose that $\left\{\mathcal{T}_{z}\right\}_{z \in C}$ is an admissible family of operators satisfying

$$
\left\|\mathcal{T}_{a+i y}\right\|_{L^{q_{a}\left(\mathbb{Q}_{p}^{N}\right)}} \leqslant M_{a}\|f\|_{L^{q_{a}}\left(\mathbb{Q}_{p}^{N}\right)}
$$

and

$$
\left\|T_{b+i y}\right\|_{L^{q_{b}\left(\mathbb{Q}_{p}^{N}\right)}} \leqslant M_{b}\|f\|_{L^{q_{b}}\left(\mathbb{Q}_{p}^{N}\right)}
$$

for all $f \in \mathcal{S}\left(\mathbb{Q}_{p}^{N}\right)$, where $1 \leqslant q_{a}, q_{b} \leqslant \infty$ and $M_{a}, M_{b}$ are constants. Then

$$
\left\|\mathcal{T}_{(b-a) \theta+a}\right\|_{L^{q \theta}\left(\mathbb{Q}_{p}^{N}\right)} \leqslant M_{a}^{1-\theta} M_{b}^{\theta}\|f\|_{L^{q \theta}\left(Q_{p}^{N}\right)}
$$

for all $f \in \mathcal{S}\left(\mathbb{Q}_{p}^{N}\right)$, where $1 / q_{\theta}=(1-\theta) / q_{a}+\theta / q_{b}$ and $0 \leqslant \theta \leqslant 1$. 
We 'linearise' $\mathcal{N}_{s}$ in order to apply this theorem. Let $x \mapsto k(x)$ be an arbitrary integer-valued function on $\mathbb{Q}_{p}^{N}$. With it we define the admissible family of operators $\mathcal{U}_{\boldsymbol{s}}$ by $\mathcal{U}_{s} f(x)=\nu_{k(x)}^{s} * f(x)$. As

$$
\mathcal{U}_{0} f(x)=\nu_{k(x)}^{0} * f(x)=\mu_{k(x)} * f(x),
$$

we let $b=1 / 2 N$ and take $\theta$ and $a$ so that $(1 / 2 N-a) \theta+a=0$. It is not hard to show that $\theta$ and $a$ can be chosen so that $q_{\theta}$ can take all the values in the range $1<q_{\theta}<\infty$. Hence we can interpolate between the bounds in Proposition 17 and Proposition 15 so that

$$
\left\|\mathcal{U}_{0} f\right\|_{L^{q}\left(\mathbb{Q}_{p}^{N}\right)} \leqslant C_{q}\|f\|_{L^{q}\left(\mathbb{Q}_{p}^{N}\right)}
$$

for all $f \in \mathcal{S}\left(\mathbb{Q}_{p}^{N}\right)$. Now as $x \mapsto k(x)$ is arbitrary, and

$$
\mathcal{N}_{0} f(x)=\sup _{k \in \mathbf{Z}} \nu_{k}^{0} * f(x)=\mathcal{M}_{\tilde{\gamma}} f(x)
$$

we obtain the following proposition.

THEOREM 19. Suppose that $1<q<\infty$ and that $\mathcal{M}_{\tilde{\gamma}}$ is defined as above. Then there is a constant $C_{q}$ so that

$$
\left\|\mathcal{M}_{\tilde{\gamma}} f\right\|_{L^{q}\left(\mathbb{Q}_{p}^{N}\right)} \leqslant C_{q}\|f\|_{L^{q}\left(\mathbb{Q}_{p}^{N}\right)}
$$

for all $f \in \mathcal{S}\left(\mathbb{Q}_{p}^{N}\right)$.

\section{THE METHOD OF DESCENT}

We shall now return to our original curve and dimension. This technique has its origins in a paper of de Leeuw [1].

THEOREM 20. Suppose that $1<q<\infty$ and that $\mathcal{M}_{\gamma}$ is defined as above. Then there is a constant $C_{q}$ so that

$$
\left\|\mathcal{M}_{\gamma} f\right\|_{L^{q}\left(\mathbb{Q}_{p}^{n}\right)} \leqslant C_{q}\|f\|_{L^{q}\left(\mathbb{Q}_{p}^{n}\right)}
$$

for all $f \in \mathcal{S}\left(\mathbb{Q}_{p}^{n}\right)$.

Proof: Let $K$ be a positive integer, and define $\mathcal{M}_{\gamma}^{K}$ by

$$
\mathcal{M}_{\gamma}^{K} f(x)=\sup _{|k| \leqslant K} \frac{1}{p^{k}} \int_{|t| \leqslant p^{k}} f(x-\gamma(t)) d t,
$$

where $k$ takes integer values, and $\mathcal{M}_{\bar{\gamma}}^{K}$ by

$$
\mathcal{M}_{\tilde{\gamma}}^{K} f(x)=\sup _{|k| \leqslant K} \frac{1}{p^{k}} \int_{|t| \leqslant p^{k}} f(x-\tilde{\gamma}(t)) d t
$$


Recall that $R: \mathbb{Q}_{p}^{N} \longrightarrow \mathbb{Q}_{p}^{n}$ is a linear map so that

$$
R\left(t, t^{2}, \ldots, t^{N}\right)=\left(P_{1}(t), \ldots, P_{n}(t)\right)
$$

and

$$
\mathcal{M}_{\gamma}^{K} f(x)=\sup _{|k| \leqslant K} \frac{1}{p^{k}} \int_{|t| \leqslant p^{k}} f(x-R(\widetilde{\gamma}(t)) d t .
$$

Let $T_{y} f(x)=f(x+R(y))$, so that

$$
\left\|\mathcal{M}_{\gamma}^{K} f\right\|_{L^{q}\left(\mathbb{Q}_{p}^{n}\right)}^{q}=\frac{1}{p^{N r}} \int_{B_{r}(0)}\left\|T_{y} \mathcal{M}_{\gamma}^{K} f\right\|_{L^{q}\left(\mathbb{Q}_{p}^{n}\right)}^{q} d y
$$

where $B_{r}(0)=\left\{y \in \mathbb{Q}_{p}^{N}:|y| \leqslant p^{r}\right\}$. At this point $K$ is fixed, so we may choose $r$ so that $r>N K$. If $y \in B_{r}(0)$ and $|t| \leqslant p^{k}$, then $|y-\widetilde{\gamma}(t)| \leqslant p^{r}$. Hence, when $y \in B_{r}(0)$,

$$
\begin{aligned}
T_{y} \mathcal{M}_{\gamma}^{K} f(x) & =\sup _{|k| \leqslant K} \frac{1}{p^{k}} \int_{|t| \leqslant p^{k}} f(x+R(y-\tilde{\gamma}(t))) d t \\
& =\sup _{|k| \leqslant K} \frac{1}{p^{k}} \int_{|t| \leqslant p^{k}} f(x+R(y-\widetilde{\gamma}(t))) 1_{r}(y-\widetilde{\gamma}(t)) d t,
\end{aligned}
$$

where $1_{r}$ denotes the characteristic function of

$$
B_{r}(0)=\left\{y \in \mathbb{Q}_{p}^{N}:|y| \leqslant p^{r}\right\}
$$

Thus,

$$
T_{y} \mathcal{M}_{\gamma}^{K} f(x)=\sup _{|k| \leqslant K} \frac{1}{p^{k}} \int_{|t| \leqslant p^{k}} F_{x}(y-\widetilde{\gamma}(t)) d t=\mathcal{M}_{\tilde{\gamma}}^{K} F_{x}(y),
$$

where $F_{x}(z)=f(x+R(z)) 1_{r}(z)$.

We have

$$
\int_{B_{r}(0)}\left|\mathcal{M}_{\widetilde{\gamma}}^{K} F_{x}(y)\right|^{q} d y \leqslant C_{q}^{q} \int_{\mathbb{Q}_{p}^{N}}\left|F_{x}(y)\right|^{q} d y,
$$

by Theorem 19, and by Fubini's theorem,

$$
\int_{\mathbb{Q}_{p}^{n}} \int_{\mathbb{Q}_{p}^{N}}\left|F_{x}(y)\right|^{q} d y d x=p^{N r}\|f\|_{L^{q}\left(\mathbb{Q}_{p}^{n}\right)}^{q} .
$$

Hence we can integrate (9) to obtain

$$
\int_{\mathbb{Q}_{p}^{n}} \int_{B_{r}(0)}\left|\mathcal{M}_{\tilde{\gamma}}^{K} F_{x}(y)\right|^{q} d y d x \leqslant p^{N r} C_{q}^{q}\|f\|_{L^{q}\left(\mathbb{Q}_{p}^{n}\right)}^{q} .
$$

Now we combine $(7),(8)$ and $(10)$ so that

$$
\left\|\mathcal{M}_{\gamma}^{K} f\right\|_{L^{q}\left(\mathbb{Q}_{p}^{n}\right)}^{q} \leqslant \frac{p^{N r}}{p^{N r}} C_{q}^{q}\|f\|_{L^{q}\left(\mathbb{Q}_{p}^{n}\right)}^{q}=C_{q}^{q}\|f\|_{L^{q}\left(\mathbb{Q}_{p}^{n}\right)}^{q},
$$

and let $K$ tend to infinity to obtain the result. 


\section{CONCLUSION}

As the space of compactly supported, locally constant functions $\mathcal{S}\left(\mathbb{Q}_{p}^{n}\right)$ is dense in $L^{q}\left(\mathbb{Q}_{p}^{n}\right)$, when $1 \leqslant q<\infty$, it is clear that Theorem 1 can be obtained from Theorem 20 by a simple limiting argument. We note the following corollary, which is a version of the fundamental theorem of calculus along $p$-adic curves. The proof is the same as that for Corollary 12 .

COROLlaRY 21. Suppose that $f \in L^{q}\left(\mathbb{Q}_{p}^{n}\right)$, where $1<q<\infty$. Suppose that $\gamma$ is defined as above, with $\gamma(0)=0$. Then for almost every $x \in \mathbb{Q}_{p}^{n}$,

$$
\lim _{k \rightarrow-\infty} \frac{1}{p^{k}} \int_{|t| \leqslant p^{k}} f(x-\gamma(t)) d t=f(x)
$$

It is an open question, in both the Euclidean and $p$-adic cases, as to whether there is a weak type $(1,1)$ version of Theorem 1 . Similarly, it is not known whether there is an $L^{1}$ version of Corollary 21.

\section{REFERENCES}

[1] K. de Leeuw, 'On $L_{p}$ multipliers', Ann. of Math. 81 (1965), 364-379.

[2] K.M. Rogers, 'A van der Corput lemma for the p-adic numbers', Proc. Amer. Math. Soc. (to appear).

[3] W.H. Schikhof, Ultrametric calculus (Cambridge Univ. Press, Cambridge, 1984).

[4] E.M. Stein, Harmonic analysis: real-variable methods, orthogonality, and oscillatory integrals (Princeton Univ. Press, Princeton, NJ, 1993).

[5] E.M. Stein and S. Wainger, 'Problems in harmonic analysis related to curvature', Bull. Amer. Math. Soc. 84 (1978), 1239-1295.

[6] E.M. Stein and G. Weiss, Introduction to Fourier analysis on Euclidean spaces (Princeton Univ. Press, Princeton, N.J. 1971).

[7] M.H. Taibleson, 'Harmonic analysis on $n$-dimensional vector spaces over local fields, I. Basic results on fractional integration', Math. Ann. 176 (1968), 191-207.

[8] M.H. Taibleson, Fourier analysis on local fields (Princeton Univ. Press, Princeton, N.J., 1975).

School of Mathematics

University of New South Wales

Sydney, NSW 2052

Australia

e-mail: K.M.Rogers.99@cantab.net 